\title{
Transmission Rate of Remote Monitoring and Mortality in Patients With Pacemaker
}

\author{
Toshihiko Goto, MD; Kento Mori, MD; Takafumi Nakayama, MD; Junki Yamamoto, MD; \\ Yasuhiro Shintani, MD; Kosuke Nakasuka, MD; Kazuaki Wakami, MD; \\ Hidekatsu Fukuta, MD; Yoshihiro Seo, MD; Nobuyuki Ohte, MD
}

\begin{abstract}
Background: Remote monitoring of cardiac implantable electronic devices improves clinical outcomes, but data on the association between the transmission rate (TR) of the remote monitoring, calculated in percentage as the ratio between days of transmission and days of follow-up after remote monitoring introduction, and death in patients with a pacemaker are limited.

Methods and Results: In this single-center retrospective observational study, we investigated 180 patients with a newly implanted pacemaker capable of using a specific remote monitoring system with daily transmission (79.5 \pm 8.8 years, men $50.6 \%)$. The study endpoint was all-cause death. During the follow-up period (median 2.7 years), 33 all-cause deaths were reported, and the TR was significantly lower in the deceased patients than in the survivors $(89.6 \pm 9.6 \%$ vs. $95.4 \pm 7.0 \%, P<0.001)$. The area under the receiveroperating characteristic curve for TR to predict all-cause death was 0.72 (95\% confidence interval $[\mathrm{Cl}] 0.62-0.81, \mathrm{P}<0.001)$. A TR of $95 \%$ had sensitivity of $74.1 \%$ and specificity of $63.6 \%$ for predicting all-cause death. In the multivariate Cox regression analysis, TR $<95 \%$ was selected as a predictor of all-cause death (hazard ratio $3.43,95 \% \mathrm{Cl} 1.61-7.27, \mathrm{P}=0.001$ ).
\end{abstract}

Conclusions: Low TR is a predictor of all-cause death in patients with a pacemaker. Patients with TR $295 \%$ may experience a lower incidence of death, and should have a good prognosis.

Key Words: Arrhythmia; Pacemakers; Prognosis; Remote monitoring; Transmission rate

$\mathbf{R}$ emote monitoring of cardiac implantable electronic devices (CIEDs) has been accepted as the standard of care for the follow-up of patients with CIEDs. ${ }^{1}$ Several large, randomized prospective trials have consistently shown that both remote monitoring and remote interrogation can provide earlier detection of actionable events and do not compromise device safety or patients' health compared with traditional follow-up visits for device check-up. ${ }^{24}$ Furthermore, some studies have reported that remote monitoring improves clinical outcomes in patients with implantable cardioverter-defibrillators (ICD), cardiac resynchronization therapy-pacing (CRT-P), or cardiac resynchronization therapy-defibrillators (CRT-D). ${ }^{\mathbf{5 6}}$ As remote monitoring management requires data transmission, patients must be connected to a remote monitoring system. Although different types of such systems exist, a particular remote monitoring system can allow patients to daily transmit data automatically by plugging the transmitter into an outlet. However, data about the relationship between the transmission rate (TR) of remote monitoring and death in patients with a pacemaker are still limited. Thus, the aims of this study were to assess this association between TR and prognosis in patients with a pacemaker, and to investigate whether TR could predict all-cause death in such patients.

Methods
Study Patients
This was a single-center retrospective observational study.
A total of 278 consecutive patients with a newly implanted
pacemaker for standard pacing indications between August
2011 and December 2018 were investigated at Nagoya City
University Hospital. Patients were eligible for inclusion in
this study if (1) they had undergone implantation of a
pacemaker capable of using a specific remote monitoring
system with daily transmission (Home Monitoring
Biotronik SE and Co. KG, Berlin, Germany) to calculate
TR and their TR was $\geq 60 \%$, and (2) they were followed up

Received June 30, 2020; accepted June 30, 2020; J-STAGE Advance Publication released online August 29, 2020 Time for primary review: 1 day

Department of Cardiology, Nagoya City University Graduate School of Medical Sciences, Nagoya (T.G., K.M., T.N., J.Y., Y. Shintani, K.N., K.W., Y. Seo, N.O.); Clinical Research Management Center, Nagoya City University Hospital, Nagoya (H.F.), Japan

Y. Seo is a member of Circulation Reports' Editorial Team.

Mailing address: Toshihiko Goto, MD, Department of Cardiology, Nagoya City University Graduate School of Medical Sciences, Mizuho-cho, Mizuho-ku, Nagoya 467-8601, Japan. E-mail: t-goto@med.nagoya-cu.ac.jp

All rights are reserved to the Japanese Circulation Society. For permissions, please e-mail: cr@j-circ.or.jp

ISSN-2434-0790

Circulation Reports Vol.2, September 2020 


\begin{tabular}{|c|c|c|c|c|c|c|c|}
\hline Characteristic & $\begin{array}{c}\text { All } \\
\text { patients } \\
(n=180)\end{array}$ & $\begin{array}{l}\text { Without } \\
\text { events }{ }^{*} \\
(n=147)\end{array}$ & $\begin{array}{l}\text { With } \\
\text { events* } \\
(n=33)\end{array}$ & $P$ value & $\begin{array}{l}\text { Without } \\
\text { events }{ }^{\star *} \\
(n=137)\end{array}$ & $\begin{array}{c}\text { With } \\
\text { events } \\
(n=43)\end{array}$ & $P$ value \\
\hline Male/female & $91 / 89$ & $73 / 74$ & $18 / 15$ & 0.61 & $67 / 70$ & $24 / 19$ & 0.43 \\
\hline Age (years) & $79.5 \pm 8.8$ & $78.5 \pm 8.8$ & $83.9 \pm 7.6$ & 0.001 & $78.2 \pm 8.6$ & $83.7 \pm 8.1$ & $<0.001$ \\
\hline Height (cm) & $155.6 \pm 10.0$ & $156.2 \pm 9.8$ & $152.8 \pm 10.5$ & 0.08 & $156.3 \pm 9.7$ & $153.2 \pm 10.7$ & 0.07 \\
\hline Weight (kg) & $54.7 \pm 11.2$ & $55.3 \pm 10.9$ & $51.9 \pm 12.5$ & 0.11 & $55.5 \pm 10.7$ & $52.3 \pm 12.5$ & 0.10 \\
\hline Body mass index $\left(\mathrm{kg} / \mathrm{m}^{2}\right)$ & $22.5 \pm 3.5$ & $22.6 \pm 3.3$ & $22.1 \pm 4.2$ & 0.42 & $22.6 \pm 3.2$ & $22.1 \pm 4.2$ & 0.44 \\
\hline \multicolumn{8}{|l|}{ Echocardiographic parameters } \\
\hline LV ejection fraction (\%) & $67.8 \pm 11.8$ & $67.9 \pm 11.9$ & $67.5 \pm 11.8$ & 0.85 & $68.6 \pm 11.6$ & $65.5 \pm 12.3$ & 0.13 \\
\hline $\mathrm{LAD}(\mathrm{mm})$ & $40.8 \pm 6.3$ & $40.8 \pm 6.2$ & $41.1 \pm 6.6$ & 0.80 & $40.8 \pm 6.3$ & $40.9 \pm 6.1$ & 0.93 \\
\hline \multicolumn{8}{|l|}{ Indication for pacemaker implantation } \\
\hline SSS/AVB & $80 / 100$ & $70 / 77$ & $10 / 23$ & 0.07 & $61 / 76$ & $19 / 24$ & 0.97 \\
\hline \multicolumn{8}{|l|}{ Pacemaker parameters } \\
\hline \multicolumn{8}{|l|}{ Right atrial pacing site } \\
\hline Appendage/low septum & $163 / 4$ & $135 / 4$ & $28 / 0$ & 0.36 & $126 / 4$ & $37 / 0$ & 0.28 \\
\hline \multicolumn{8}{|l|}{ Right ventricular pacing site } \\
\hline Low septum/apex & $83 / 97$ & $68 / 79$ & $15 / 18$ & 0.93 & $62 / 75$ & $21 / 22$ & 0.68 \\
\hline Pacing rate (beats/min) & $57.2 \pm 5.7$ & $56.8 \pm 5.7$ & $58.8 \pm 5.5$ & 0.07 & $56.8 \pm 5.4$ & $58.4 \pm 6.5$ & 0.11 \\
\hline Paced QRS intervals (mm) & $147.8 \pm 17.1$ & $147.2 \pm 17.0$ & $150.2 \pm 17.4$ & 0.37 & $147.0 \pm 17.5$ & $150.2 \pm 17.8$ & 0.29 \\
\hline Physiologic/ventricular pacing & $163 / 17$ & $137 / 10$ & $26 / 7$ & 0.01 & $128 / 9$ & $35 / 8$ & 0.02 \\
\hline Right ventricular pacing >40\% (\%) & 56.1 & 53.1 & 69.7 & 0.08 & 54.7 & 60.5 & 0.51 \\
\hline Living alone (\%) & 13.3 & 12.2 & 18.2 & 0.37 & 13.1 & 14.0 & 0.89 \\
\hline Being below the poverty line (\%) & 3.9 & 4.1 & 3.0 & 0.78 & 3.6 & 4.7 & 0.77 \\
\hline Length of hospital stay (day) & $17.3 \pm 11.5$ & $16.3 \pm 11.2$ & $21.8 \pm 12.0$ & 0.01 & $16.7 \pm 11.9$ & $19.3 \pm 10.2$ & 0.21 \\
\hline Barthel index & $92.5 \pm 21.8$ & $94.3 \pm 19.9$ & $84.2 \pm 27.6$ & 0.02 & $93.9 \pm 20.6$ & $87.9 \pm 25.0$ & 0.12 \\
\hline Transmission rate $(\%)^{*}$ & $94.4 \pm 7.9$ & $95.4 \pm 7.0$ & $89.6 \pm 9.6$ & $<0.001$ & & & \\
\hline Transmission rate $(\%)^{\star \star}$ & $93.6 \pm 9.6$ & & & & $95.5 \pm 7.0$ & $90.4 \pm 10.1$ & $<0.001$ \\
\hline
\end{tabular}

Data are expressed as mean \pm standard deviation or number or frequency (\%). ${ }^{*}$ Events defined as all-cause death. ${ }^{* *}$ Events defined as a composite of all-cause death and first heart failure hospitalization. AVB, atrioventricular block; LAD, left atrial dimension; LV, left ventricular; SSS, sick sinus syndrome.

for $\geq 6$ months. Patients who disagreed with using the remote monitoring system were excluded. No patients withdrew their consent during the study period.

\section{Data Collection}

We investigated the patients' baseline characteristics, comprehensive echocardiographic indices, underlying diseases, plasma B-type natriuretic peptide (BNP) levels, estimated glomerular filtration rate (eGFR), indication for pacemaker implantation, pacing mode [physiological pacing: $\mathrm{DDD}(\mathrm{R}), \mathrm{VDD}(\mathrm{R})$; or ventricular pacing: $\mathrm{VVI}(\mathrm{R})$, $\mathrm{DDI}(\mathrm{R})]$, and Barthel index (BI) from their medical records. Plasma BNP level, eGFR, echocardiographic indices, and $\mathrm{BI}$ were collected at the time of discharge.

\section{Outcome Measures}

The BI consists of 10 items: feeding, bathing, grooming, dressing, bowel control, bladder control, toileting, chair transfer, ambulation, and stair climbing. This is widely used as an indicator of activities of daily living (ADLs) in routine clinical practice in geriatric medicine (BI range, 0-100; BI $\geq 85$ indicates independent ADLs). ${ }^{7}$ The Charlson comorbidity index (CCI), ${ }^{8}$ which is a method for predicting death by classifying or weighting comorbidities, was also measured. The severity of comorbidities was categorized by CCI score, as follows: mild, CCI score 1-2; moderate, CCI score 3-4; and severe, CCI score $\geq 5$. The definition of each underlying disease was derived from the CCI criteria. ${ }^{8}$
Hypertension was defined as systolic blood pressure $\geq 140 \mathrm{mmHg}$ and/or diastolic blood pressure $\geq 90 \mathrm{mmHg}$ or current treatment with antihypertensive drugs. The study endpoints were all-cause death and a composite of all-cause death and first heart failure hospitalization.

The indications for pacemaker implantation included sick sinus syndrome $(\mathrm{n}=80)$ and atrioventricular block $(n=100)$. A total of 163 atrial leads were placed in the right atrial appendage, and 4 were placed in the atrial septum. Similarly, 83 ventricular leads were placed in the lower right ventricular septum and 97 were placed in the right ventricular apex. Patients were followed by remote monitoring in addition to follow-up visits for device interrogation every 3-12 months. The study protocol was approved by Nagoya City University Graduate School of Medical Sciences and Nagoya City University Hospital Institutional Review Board (reference no. 60-19-0172), and was carried out in accordance with the Declaration of Helsinki.

\section{Remote Monitoring and TR}

Each patient received a commercially available pacemaker (Evia, Etrinsa, Eluna, Edora; Biotronik SE \& Co.) capable of using Home Monitoring, which is a remote monitoring system that automatically transmits data stored in implantable devices to Biotronik service center over a wireless and global system via mobile communications network at a set time every day (typically at 04:00 hours). The individual percentage of right ventricular pacing was obtained from 


\begin{tabular}{|c|c|c|c|c|c|c|c|}
\hline Characteristic & $\begin{array}{c}\text { All } \\
\text { patients } \\
(n=180)\end{array}$ & $\begin{array}{l}\text { Without } \\
\text { events* } \\
\text { (n=147) }\end{array}$ & $\begin{array}{c}\text { With } \\
\text { events* } \\
(n=33)\end{array}$ & $P$ value & $\begin{array}{l}\text { Without } \\
\text { events } \\
(n=137)\end{array}$ & $\begin{array}{c}\text { With } \\
\text { events } \\
(n=43)\end{array}$ & $P$ value \\
\hline \multicolumn{8}{|l|}{ Underlying disease } \\
\hline Hypertension (\%) & 68.3 & 66.7 & 75.8 & 0.31 & 67.2 & 72.1 & 0.54 \\
\hline Diabetes mellitus (\%) & 20.6 & 19.0 & 27.3 & 0.29 & 18.2 & 27.9 & 0.17 \\
\hline $\begin{array}{l}\text { Diabetes mellitus with organ } \\
\text { damage }(\%)\end{array}$ & 11.1 & 8.2 & 24.2 & 0.008 & 8.0 & 20.9 & 0.02 \\
\hline Prior history of MI (\%) & 8.9 & 6.1 & 21.2 & 0.006 & 5.1 & 20.9 & 0.001 \\
\hline Heart failure (\%) & 38.3 & 33.3 & 60.6 & 0.004 & 29.9 & 65.1 & $<0.001$ \\
\hline Prior history of stroke (\%) & 9.4 & 7.5 & 18.2 & 0.06 & 6.6 & 18.6 & 0.02 \\
\hline Hemiplegia (\%) & 2.2 & 1.4 & 6.1 & 0.10 & 2.2 & 2.3 & 0.96 \\
\hline Dementia or Alzheimer's (\%) & 16.7 & 14.3 & 27.3 & 0.07 & 14.6 & 23.3 & 0.18 \\
\hline Peripheral arterial disease (\%) & 5.0 & 2.0 & 18.2 & $<0.001$ & 2.2 & 14.0 & 0.002 \\
\hline Pulmonary disease/asthma (\%) & 7.2 & 8.2 & 3.0 & 0.30 & 8.8 & 2.3 & 0.16 \\
\hline Moderate/severe renal disease (\%) & 3.9 & 1.4 & 15.2 & $<0.001$ & 2.2 & 9.3 & 0.04 \\
\hline $\begin{array}{l}\text { Rheumatic or connective tissue } \\
\text { disease (\%) }\end{array}$ & 2.8 & 2.7 & 3.0 & 0.92 & 2.9 & 2.3 & 0.84 \\
\hline Gastric or peptic ulcer (\%) & 2.2 & 2.7 & 0.0 & 0.34 & 2.9 & 0.0 & 0.26 \\
\hline Chronic liver disease (\%) & 2.8 & 2.7 & 3.0 & 0.92 & 2.9 & 2.3 & 0.84 \\
\hline Solid cancer (\%) & 15.6 & 12.9 & 27.3 & 0.04 & 13.1 & 23.3 & 0.11 \\
\hline Lymphoma (\%) & 1.7 & 2.0 & 0.0 & 0.41 & 2.2 & 0.0 & 0.33 \\
\hline Charlson comorbidity index & $1.8 \pm 1.8$ & $1.5 \pm 1.5$ & $3.3 \pm 2.2$ & $<0.001$ & $1.5 \pm 1.5$ & $2.9 \pm 2.1$ & $<0.001$ \\
\hline \multicolumn{8}{|l|}{ Laboratory data } \\
\hline $\mathrm{BNP}(\mathrm{mg} / \mathrm{dL})$ & $\begin{array}{c}99.8 \text { [IQR, } \\
51.0-216.0]\end{array}$ & $\begin{array}{c}91.4 \text { [IQR, } \\
45.0-199.7]\end{array}$ & $\begin{array}{c}199.7[\mathrm{IQR}, \\
107.8-314.1]\end{array}$ & 0.006 & $\begin{array}{l}83.2 \text { [IQR, } \\
44.3-199.7]\end{array}$ & $\begin{array}{l}199.7[\mathrm{IQR} \\
99.6-358.0]\end{array}$ & 0.001 \\
\hline $\mathrm{HbA1c}(\%)$ & $6.2 \pm 0.8$ & $6.1 \pm 0.8$ & $6.3 \pm 0.8$ & 0.30 & $6.2 \pm 0.8$ & $6.3 \pm 0.7$ & 0.40 \\
\hline Serum creatinine $(\mathrm{mg} / \mathrm{dL})$ & $1.4 \pm 3.4$ & $1.2 \pm 3.7$ & $1.9 \pm 2.2$ & 0.34 & $1.3 \pm 3.8$ & $1.6 \pm 1.9$ & 0.62 \\
\hline eGFR $\left(\mathrm{mL} / \mathrm{min} / 1.73 \mathrm{~m}^{2}\right)$ & $61.0 \pm 24.9$ & $64.1 \pm 22.5$ & $47.3 \pm 30.3$ & $<0.001$ & $63.9 \pm 22.8$ & $51.8 \pm 29.3$ & 0.005 \\
\hline \multicolumn{8}{|l|}{ Medications } \\
\hline Diuretic (\%) & 24.4 & 19.0 & 48.5 & $<0.001$ & 17.5 & 46.5 & $<0.001$ \\
\hline Statin (\%) & 26.1 & 25.2 & 30.3 & 0.54 & 25.5 & 27.9 & 0.76 \\
\hline ACEI (\%) & 7.8 & 8.2 & 6.1 & 0.68 & 8.8 & 4.7 & 0.38 \\
\hline ARB (\%) & 40.6 & 40.8 & 39.4 & 0.88 & 40.9 & 39.5 & 0.88 \\
\hline$\beta$-blocker (\%) & 15.6 & 15.0 & 18.2 & 0.65 & 14.6 & 18.6 & 0.53 \\
\hline CCB (\%) & 42.2 & 38.8 & 57.6 & 0.05 & 38.7 & 53.5 & 0.09 \\
\hline
\end{tabular}

Data are expressed as mean \pm standard deviation or number or frequency (\%). BNP is presented as median and interquartile range (IQR). ${ }^{\star}$ Events defined as all-cause death. ${ }^{* *}$ Events defined as a composite of all-cause death and first heart failure hospitalization. ACEI, angiotensinconverting enzyme inhibitor; ARB, angiotensin-receptor blocker; BNP, B-type natriuretic peptide; CCB, calcium-channel blocker; eGFR, estimated glomerular filtration rate; MI, myocardial infarction.

Home Monitoring and defined as the mean value for each individual during the 3-month period prior to the end of the study. The TR of remote monitoring was determined in percentage as the ratio of days of transmission divided by days of follow-up after remote monitoring introduction. As the transmitter of remote monitoring cannot be used during hospitalization, the days of hospitalization were excluded from the days of follow-up after remote monitoring introduction in the above formula.

\section{Statistical Analysis}

SPSS version 23.0 (SPSS Inc., Chicago, IL, USA) was used for all statistical analyses. Continuous variables are presented as mean \pm standard deviation for normally distributed variables and median and interquartile range (IQR) for non-normally distributed variables. Categorical variables are summarized as frequencies (\%). For the comparison of 2 groups, continuous variables were compared by unpaired Student's t-tests for normally distributed variables and Mann-Whitney U-tests for non-normally distributed variables, respectively. Differences in prevalence between groups were compared using the chi-square test. The ability of TR to identify death was evaluated with receiver-operating characteristic (ROC) curve analysis, where the area under the curve and $95 \%$ confidence intervals (CIs) were calculated. For endpoint-free survival analysis, Kaplan-Meier curves were generated and compared using the log-rank test. We calculated hazard ratios (HRs) derived from the Cox regression analysis to identify predictors of all-cause death. The variables that were significantly different between the deceased patients and survivors were entered into the Cox analysis as follows: age $\geq 80$ years, ventricular pacing mode, $\mathrm{BI}<85, \mathrm{CCI} \geq 3$, and $\mathrm{TR}<95 \%$. Age $\geq 80$ years, ventricular pacing mode, CCI $\geq 3$, and $\mathrm{TR}<95 \%$ were entered in the Cox regression model, but not $\mathrm{BI}<85$, in the analysis with all-cause death and heart failure hospitalization as the endpoint, because no significant difference was found in BI between patients who did or did not reach 
A.

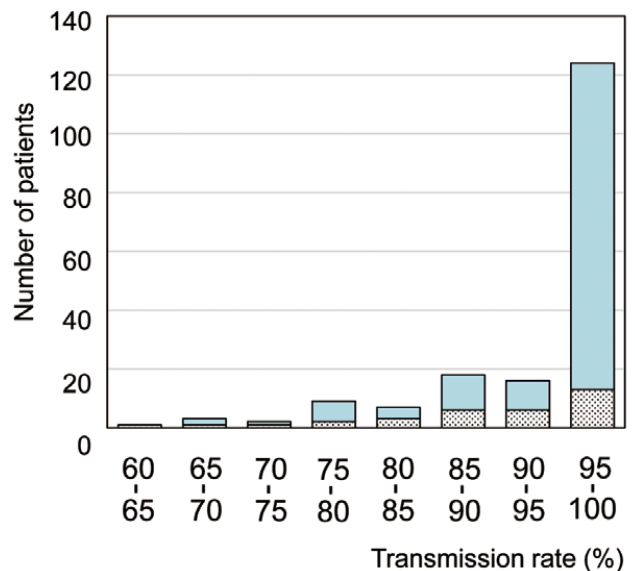

Without mortality events

With mortality events
B.

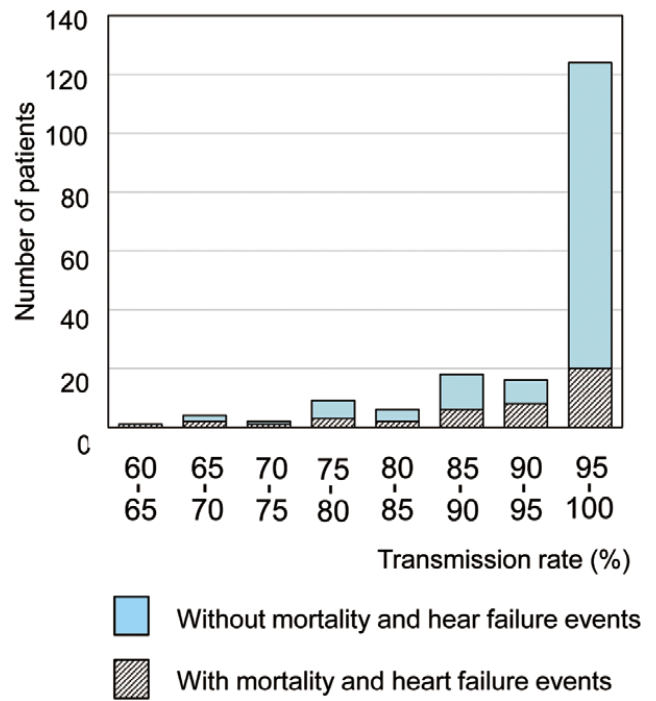

Figure 1. Distribution of transmission rate (TR). The event is death (A) or a composite of death and heart failure hospitalization (B). The distribution of patients for both events is low on the righthand side, which indicates higher range of TR.

A.

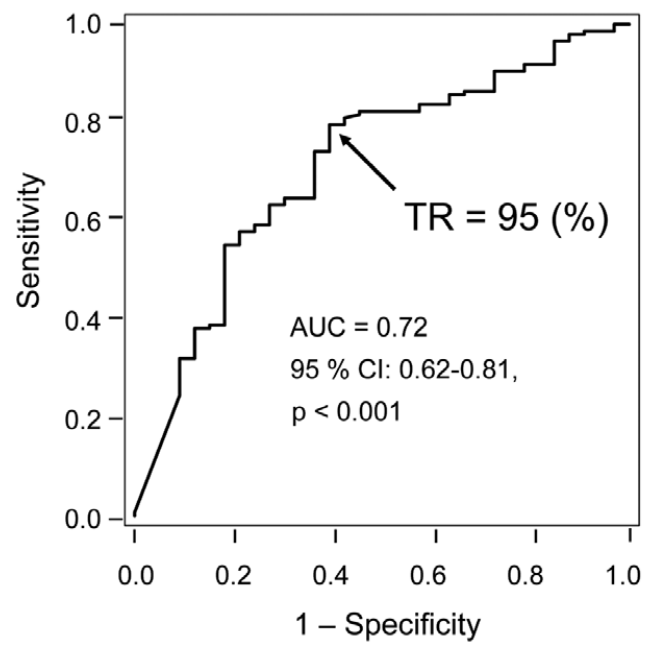

B.

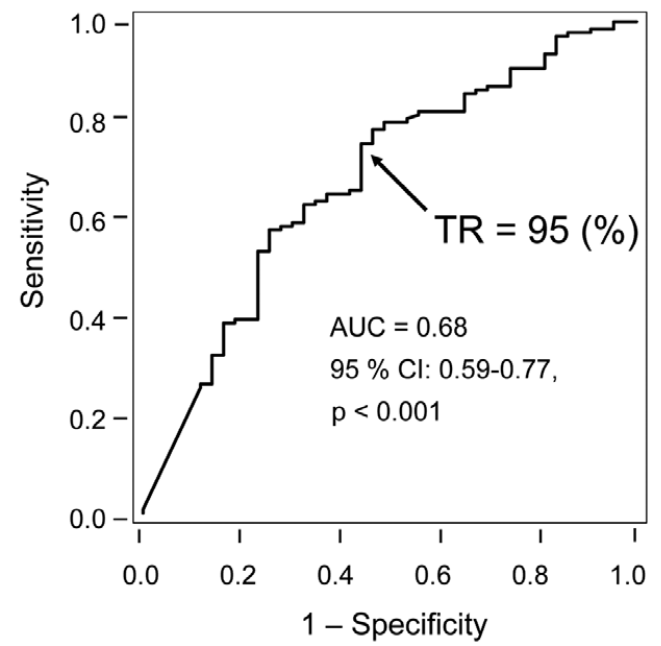

Figure 2. Receiver-operating characteristic $(R O C)$ curves for transmission rate (TR) to predict all-cause death $(\mathbf{A})$ and a composite of all-cause death and hear failure hospitalization (B). The area under the ROC (ROC) curve (AUC) for TR to predict all-cause death was 0.72 (95\% confidence interval $[\mathrm{Cl}] 0.62-0.81, \mathrm{P}<0.001)$. A TR of $95 \%$ had a sensitivity of $74.1 \%$ and a specificity of $63.6 \%$ for predicting all-cause death $(\mathbf{A})$. On the other hand, the area under the ROC curve for TR to predict a composite of all-cause death and heart failure hospitalization was 0.68 (95\% Cl 0.59-0.77, P<0.001). A TR of 95\% has sensitivity and specificity of $75.2 \%$ and $55.8 \%$, respectively, for predicting the composite outcome (B).

the endpoints. As there were too many factors with regard to the underlying disease, $\mathrm{CCI}$ was used as a representative variable. The value of age was derived from the approximate median values of the study patients. Differences with a $P$-value $<0.05$ were considered statistically significant.

\section{Results}

A total of 180 patients were included in the final analysis (age $79.5 \pm 8.8$ years, men $50.6 \%$ ). The clinical characteristics of all patients and subgroup demographics are shown in Table 1. 
A.

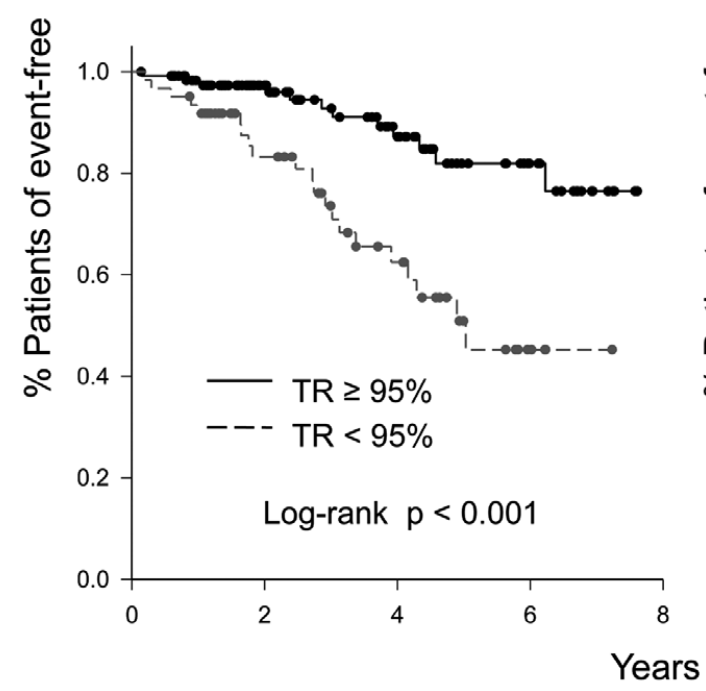

B.

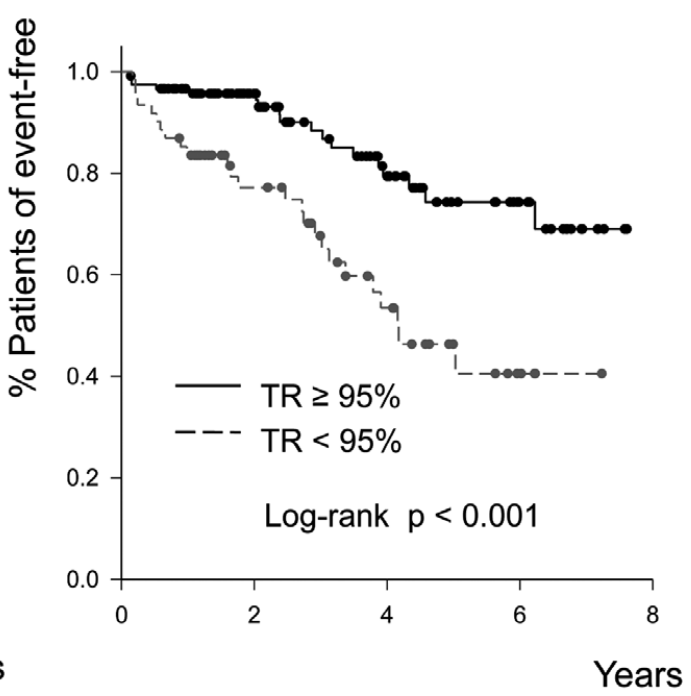

Figure 3. Kaplan-Meier curves for all-cause death $(\mathbf{A})$ and a composite of all-cause death and heart failure hospitalization (B) in patients with transmission rate (TR) $\geq 95 \%$. In both cases, the endpoint-free survival rate was significantly higher in patients with TR $\geq 95 \%$ than in those with TR $<95 \%$.

During the follow-up period (median 2.7 years, mean $3.2 \pm 2.0$ years), 33 all-cause deaths were reported. The causes of death were senility $(n=9)$, infection $(n=8)$, malignant neoplasm $(n=6)$, heart failure $(n=3)$, renal failure $(n=3)$, sudden death $(n=2)$, intestinal hemorrhage $(n=1)$, and trauma $(\mathrm{n}=1)$. Compared with patients who survived during the follow-up period, the patients who died were significantly older $(83.9 \pm 7.6$ vs. $78.5 \pm 8.8$ years, $\mathrm{P}=0.001)$. Indications for pacemaker implantation did not differ between the groups, but a trend of higher prevalence of atrioventricular block in the deceased patients than in the survivors was observed $(69.7 \%$ vs. $52.4 \%, \mathrm{P}=0.07)$. Similarly, the prevalence of patients with right ventricular pacing rate $\geq 40 \%$, a threshold value that increases the risk of heart failure, ${ }^{9}$ was also higher in the deceased patients than in the survivors $(69.7 \%$ vs. $53.1 \%, \mathrm{P}=0.08)$. Finally, the frequency of ventricular pacing mode [VVI(R) or $\mathrm{DDI}(\mathrm{R})$ mode] was higher in deceased patients than in the survivors $(21.2 \%$ vs. $6.8 \%, \mathrm{P}=0.01)$. One postoperative subcutaneous hematoma was found in this study. One lead revision was needed, and 2 cardiovascular disease treatments (elective surgery for an abdominal aneurysm; endovascular treatment of a lower limb) occurred during the follow-up period.

The proportion of diseases was significantly higher in the deceased patients than in the survivors (Table 2). As a result, the deceased patients had significantly higher CCI than the survivors $(3.3 \pm 2.2$ vs. $1.5 \pm 1.5, \mathrm{P}<0.001)$. Patients who died had significantly lower eGFR than those who survived $\left(47.3 \pm 30.3\right.$ vs. $\left.64.1 \pm 22.5 \mathrm{~mL} / \mathrm{min} / 1.73 \mathrm{~m}^{2}, \mathrm{P}<0.001\right)$. Further, plasma BNP levels in deceased patients were significantly higher than in the survivors (199.7 [IQR, 107.8-314.1] vs. 91.4 [IQR, 45.0-199.7] $\mathrm{pg} / \mathrm{mL}, \mathrm{P}=0.006$ ), which reflected the high prevalence of prior heart failure and myocardial infarction. As a reflection of cardiac function and renal function, the use of diuretics was significantly higher in deceased patients than in survivors $(48.5 \%$ vs. $19.0 \%, \mathrm{P}<0.001)$. However, despite the decline in cardiac and renal function in deceased patients, neither heart nor renal failure was a common cause of death. Increased use of calcium-channel blockers (CCBs) in deceased patients was observed. The deceased also experienced significantly longer hospital stay ( $21.8 \pm 12.0$ vs. $16.3 \pm 11.2$ days, $\mathrm{P}=0.01)$. The BI at the time of discharge was significantly lower in deceased patients than in survivors ( $84.2 \pm 27.6$ vs. $94.9 \pm 19.9$, $\mathrm{P}=0.02$ ). These results indicated that the deceased group included more patients in poorer health. In contrast, no differences in the ratio of patients who were living alone or those who were receiving welfare assistance due to poverty were found between the groups.

The TR was significantly lower in the deceased patients than in the survivors $(89.6 \pm 9.6 \%$ vs. $95.4 \pm 7.0 \%, \mathrm{P}<0.001)$. Figure 1A shows the distribution of the TR. Although most of the patients' TR was within the $95-100 \%$ range, the proportion of deceased patients in this range was low. Of the 33 deceased patients, the TR in 5 patients who died from cardiovascular death ( 3 heart failure, 2 sudden deaths) were significantly higher than in the other 28 patients who died from other causes $(98.1 \pm 3.6 \%$ vs. $88.1 \pm 9.5 \%, \mathrm{P}=0.03)$. Of the deceased patients 19 had a TR $\geq 90 \%$ (3 patients had a TR of $100 \%$ ). In the remaining 14 patients with a TR $<90 \%$, the mean TR in the initial 3 months after the introduction of remote monitoring and in the last 3 months prior to the death was significantly different $(89.0 \pm 14.7 \%$ and $64.8 \pm 23.7 \%$, respectively, $\mathrm{P}=0.02$ ) (Supplementary Figure). Therefore, the TR in about half of the deceased patients was not low from the beginning of the study. The area under the ROC curve for the TR to predict all-cause death was $0.72(95 \%$ CI $0.62-0.81, \mathrm{P}<0.001)$. From this analysis, a TR of $95 \%$ had sensitivity and specificity of $74.1 \%$ and $63.6 \%$, respectively, for predicting all-cause death (Figure 2A). The Kaplan-Meier plot showed that the 
Table 3. (A) Cross Table of TR and $\mathrm{CCl}$ Showing Number (\%) of All-Cause Deaths, (B) Cross Table of TR and CCI Showing Number (\%) of All-Cause Deaths and First Heart Failure Hospitalizations

\begin{tabular}{lcr} 
& $\mathbf{C C l}<3$ & \multicolumn{1}{c}{$\mathbf{C C l} \geq 3$} \\
(A) & & \\
$\mathrm{TR} \geq 95 \%$ & $3(3.3 \%)$ & $9(31.0 \%)$ \\
$\mathrm{TR}<95 \%$ & $11(28.2 \%)$ & $10(45.5 \%)$ \\
(B) & & \\
$\mathrm{TR} \geq 95 \%$ & $7(7.8 \%)$ & $11(37.9 \%)$ \\
$\mathrm{TR}<95 \%$ & $15(38.5 \%)$ & $10(45.5 \%)$ \\
\hline
\end{tabular}

$\mathrm{CCl}$, Charlson comorbidity index; TR, transmission rate.

incidence of all-cause death was significantly higher among those with TR $<95 \%$ than among those with TR $\geq 95 \%$ (Kaplan-Meier analysis; log-rank, $\mathrm{P}<0.001$, Figure 3A). In the survival curve by Kaplan-Meier method, a difference was found in the survival rate from the early stage, and the difference in survival rate between the groups continued to increase. This suggests that patients in poorer health were included in the deceased group as previously mentioned. The cross table of TR and CCI shown in Table $\mathbf{3 A}$ lists the number (percent) of all-cause deaths. The mortality rate in the group with CCI $\geq 3$ and $\mathrm{TR}<95 \%$ was $45.5 \%$, whereas it was $3.3 \%$ in the group with CCI $<3$ and TR $\geq 95 \%$. Subsequent multivariate Cox regression analysis was performed, with the variables entered into the model as follows: age $\geq 80$ years, ventricular pacing mode, BI $<85$, $\mathrm{CCI} \geq 3$, and $\mathrm{TR}<95 \%$.

In the multivariate Cox regression analysis, TR $<95 \%$ (HR 3.43; 95\% CI 1.61-7.27; $\mathrm{P}=0.001$ ), CCI $\geq 3$ (HR 3.80; $95 \%$ CI $1.84-7.86 ; \mathrm{P}<0.001$ ), and $\mathrm{BI}<85$ (HR 2.66; $95 \% \mathrm{CI}$ $1.09-6.49 ; \mathrm{P}=0.03)$ were selected as predictors of the endpoint during the follow-up period. Although BI was identified as a predictor of all-cause death, the HR of BI $<85$ was lower than that of TR $<95 \%$ and CCI $\geq 3$. This reflects the features of $\mathrm{BI}$; if a particular level of ADLs is maintained, the BI could easily reach the maximum score, but if performance of ADLs is impaired, it is difficult to obtain a score. In contrast, age $\geq 80$ years and ventricular pacing mode were not selected (Table 4A).
All results that defined the endpoint as a composite of all-cause death and first heart failure hospitalization (43 events in total) are also shown in Tables 1,2 and 3B. During the follow-up period (median 2.5 years, mean $3.0 \pm 2.0$ years), 17 heart failure hospitalizations (including 6 subsequent heart failure deaths) occurred. No differences were found in the length of hospital stay or in the BI between the 2 groups, when the endpoint was defined as a composite of all-cause death and heart failure hospitalization (Table 1). With regard to underlying diseases and medications, significant differences were found in the prevalence of a prior history of stroke and the frequency of use of CCBs in the analysis that added heart failure as an endpoint, but otherwise showed the same trends as the analysis with death as an endpoint (Table 2). The distribution of the TR showed the similar trend as when the endpoint was death (Figure 1B). The area under the ROC curve for the TR to predict a composite of all-cause death and heart failure hospitalization was 0.68 (95\% CI $0.59-0.77, \mathrm{P}<0.001)$. A TR of $95 \%$ had sensitivity and specificity of $75.2 \%$ and $55.8 \%$, respectively, for predicting a composite of all-cause death and heart failure hospitalization (Figure 2B). Similarly, patients with both low TR and high CCI also had the highest rates of all-cause death and heart failure hospitalization (Table 3B). A Kaplan-Meier plot showed that the incidence of a composite of all-cause death and heart failure hospitalization was significantly higher among those with TR $<95 \%$ than among those with TR $\geq 95 \%$ (Kaplan-Meier analysis; log-rank, $\mathrm{P}<0.001$, Figure 3B). In the multivariate Cox regression analysis, TR $<95 \%$ (HR 2.80; 95\% CI 1.515.22; $\mathrm{P}=0.001), \mathrm{CCI} \geq 3$ (HR 2.95; 95\% CI 1.60-5.44; $\mathrm{P}=0.001$ ), and age $\geq 80$ (HR 3.03; 95\% CI 1.55-5.90; $\mathrm{P}=0.001)$ were selected as predictors of a composite of the all-cause death and heart failure hospitalization during the follow-up period. The statistical trend was generally the same as for the all-cause death endpoint, and it is noteworthy that the high TR also remained important.

\section{Discussion}

This study demonstrated the relationship between high TR and good prognosis in pacemaker patients without apparent reduction in left ventricular ejection fraction. Extended follow-up periods are already set for patients under remote

\begin{tabular}{|lcccc|}
\hline $\begin{array}{l}\text { Table 4. (A) Factors Associated With All-Cause Mortality in Multivariate Cox Regression Analysis, (B) } \\
\text { Factors Associated With All-Cause Mortality and First Heart Failure Hospitalization Events in } \\
\text { Multivariate Cox Regression Analysis }\end{array}$ & HR & $\mathbf{9 5 \%}$ Cl & P value \\
(A) & $\boldsymbol{\beta}$ & & & \\
$\mathrm{Age}>80$ years & 0.74 & 2.10 & $0.96-4.57$ & 0.06 \\
Ventricular pacing mode & -0.04 & 0.96 & $0.36-2.57$ & 0.94 \\
Barthel index $<85$ & 0.98 & 2.66 & $1.09-6.49$ & 0.03 \\
$\mathrm{CCl} \geq 3$ & 1.34 & 3.80 & $1.84-7.86$ & $<0.001$ \\
TR $<95 \%$ & 1.23 & 3.43 & $1.61-7.27$ & 0.001 \\
(B) & & & & \\
Age $>80$ years & 1.11 & 3.03 & $1.55-5.90$ & 0.001 \\
Ventricular pacing mode & 0.59 & 1.80 & $0.83-3.93$ & 0.14 \\
CCI $\geq 3$ & 1.08 & 2.95 & $1.60-5.44$ & 0.001 \\
TR $<95 \%$ & 1.03 & 2.80 & $1.51-5.22$ & 0.001 \\
\hline
\end{tabular}

$\mathrm{CCl}$, Charlson comorbidity index; $\mathrm{Cl}$, confidence interval; $\mathrm{HR}$, hazard ratio; TR, transmission rate. 
monitoring management, so without data transmission, patients not only lose the benefits of remote monitoring, but also are not being followed for longer than conventional follow-up periods. This study demonstrated that patients with TR $\geq 95 \%$ may have lower incidence rates of all-cause death and a composite of all-cause death and first heart failure hospitalization, and thus should have a good prognosis.

The terms "remote interrogation" and "remote monitoring" are often used interchangeably, but the latter is an accepted term in both cases. ${ }^{1}$ In this study, we used the Home Monitoring system, which is remote monitoring that can transmit data daily. Previous studies have reported inconsistent results for the relationship between remote monitoring and prognosis, especially in patients with ICD, CRT-P, or CRT-D.6,10 For instance, the REM-HF study, a randomized controlled trial that compared the effect of usual care with that of weekly remote interrogation on prognosis in heart failure patients implanted with an ICD, CRT-P or CRT-D, found the latter approach did not improve outcomes. ${ }^{10}$ In contrast, the IN-TIME study, which used Home Monitoring, reported significant improvement in clinical outcomes in heart failure patients with ICD or CRT-D. ${ }^{6}$ Although these 2 studies are not directly comparable, daily remote monitoring may prove more beneficial than weekly remote interrogation. Furthermore, the TR in the former study was approximately $60 \%$ throughout the study period, while that of the latter study was $85 \%$. Thus, in addition to daily remote monitoring, high TR is also important from the standpoint of improving prognosis.

Many advantages of remote monitoring in patients with a pacemaker have been reported, such as a sense of safety similar to the scheduled conventional follow-up, ${ }^{2,11}$ early detection of arrhythmias or device malfunction, ${ }^{2}$ and reduction in the number of face-to-face visits. ${ }^{2,3,11}$ However, data about prognosis and remote monitoring in patients with a pacemaker have been limited, largely because patients with a pacemaker are generally older and have less severe heart disease than those with an ICD/CRT-D, where a sufficiently high TR is required to differentiate the prognosis. To achieve this TR level, a user-friendly transmitter is essential, as used in this study, and the mean TR of $94.4 \%$ was higher than the $85 \%$ of the IN-TIME study. On the other hand, the predictive potential of remote monitoring for heart failure development has been controversial. Even the IN-TIME study, which reported an improvement in mortality rates, could not confirm the benefit of remote monitoring to avoid worsening heart failure hospitalization. ${ }^{6}$ However, our study showed that low TR was associated with a composite endpoint of all-cause death and heart failure hospitalization.

Although a TR of $100 \%$ is desirable, there are several limiting factors: (1) patients may live where it is impossible to connect to a remote monitoring system, (2) patients cannot connect to a remote monitoring system, and (3) poor adherence.

In the first instance, a patient may be hospitalized because of other diseases. However, that factor was excluded from our TR calculation, so it cannot be the reason for the low TR in this study. In the second instance, it could also be when the patient is relatively healthy, and often not within range of the remote monitoring system, such as when traveling. Although there are portable transmitters, they are often not carried by patients. However, a good prognosis would be expected in such patients even without being connected to the remote monitoring system. The mean age of the present study patients was approximately 80 years, and the number of such patients would be limited. The final possibility of low TR is poor adherence, which is related to lack of comprehension about the disease and its treatment. Patients with a pacemaker can regain their previous life early after implantation, with subjective symptoms becoming increasingly scarce, which in turn could result in negligence with regard to remote monitoring. This is not limited to remote monitoring and may be applicable to other diseases. This speculation could explain why patients with low adherence to the use of remote monitoring reached the endpoint, including heart failure hospitalization, in the present study, despite the fact that remote monitoring is a poor predictor of heart failure. This study was not designed to predict heart failure using remote monitoring nor was it designed to intervene earlier for signals of heart failure detected by remote monitoring. Therefore, the low TR is not directly responsible for the high incidence of heart failure hospitalizations, which was more likely due to low adherence to medical care, including remote monitoring.

Although we could not fully elucidate the reason for the relationship between low TR and high CCI in this study, it is probable that mortality may be higher in patients with many diseases and low TR due to low adherence. In fact, more patients died in the low TR and high CCI group than in the high TR and low CCI group. Therefore, our results indicated that patients who use remote monitoring more are generally less sick or are more compliant.

In this study, TR $<95 \%$ was independently useful for prognosis prediction, resulting from our use of user-friendly and high TR achievable remote monitoring (i.e., Home Monitoring system). From the prognostic standpoint, daily remote monitoring is practical. Contrary to our expectations, no difference was found in social aspects between groups, which could be partly because the number of patients who lived alone or were receiving welfare assistance due to poverty was limited in this study, and the minimum requirement for data transmission was simply using the Home Monitoring system.

\section{Clinical Implications}

As the relationship between low TR and poor prognosis has become clear, identifying the patients who may have a low TR in advance is required. Furthermore, achieving a higher TR is needed in the clinical setting. However, regardless of its effectiveness, remote monitoring is still unfortunately under-utilized for various patient- and system-related reasons. Therefore, the use of remote monitoring should be reconsidered in patients who do not currently use it. To solve the system-related problems, selection of user-friendly remote monitoring systems is worth considering. As for patient-related issues, adequate patient education on the importance of remote monitoring should tackle the problem, but a previous study has shown that sufficient education about the Home Monitoring system was not associated with high TR.12 Therefore, it is necessary to build a system that can alert patients whose data have not been transmitted without increasing staff workload. One possibility is to use a central monitoring unit consisting of trained nurses and physicians as used in the IN-TIME study. ${ }^{6}$ Construction and operation of the central monitoring unit will be the next issue. 


\section{Study Limitations}

There are several that should be discussed. Firstly, this was a single-center retrospective observational study that included a limited number of patients. Secondly, the effect of interventions that increase TR on all-cause death was not investigated. If patients did not present at the scheduled follow-up visit or their data were not sent through the remote monitoring system, their condition was confirmed via a phone call from medical staff. However, this was not a systematic intervention, but rather an appropriate intervention in daily clinical practice. Therefore, a prospective study with a larger number of patients is needed to confirm whether increasing TR leads to improved prognosis in patients with a pacemaker. Finally, this study consisted of patients with a pacemaker using a specific remote monitoring with automatic remote transmission technology. Thus, it is unclear whether these results can be extended to other remote management systems.

\section{Conclusions}

Low TR is a predictor of all-cause death in patients with a pacemaker. Patients with TR $\geq 95 \%$ may have a lower risk of death and a good prognosis.

\section{Disclosures}

Y. Seo is a member of Circulation Reports' Editorial Team. The other authors declare no conflicts of interest.

\section{Data Availability}

The deidentified participant data supporting the results reported in this paper will be shared, including the research protocol, by researchers approved for data use by an independent review board (or learned intermediary) identified for that purpose. The data become available from 9 to 36 months after publication. The data can be used for meta-analysis of individual participant data on a request basis. Please directly contact the corresponding author to request data sharing.

\section{IRB Information}

Nagoya City University Graduate School of Medical Sciences and Nagoya City University Hospital Institutional Review Board (reference no. 60-19-0172).

\section{References}

1. Slotwiner D, Varma N, Akar JG, Annas G, Beardsall M, Fogel RI, et al. HRS Expert Consensus Statement on remote interrogation and monitoring for cardiovascular implantable electronic devices. Heart Rhythm 2015; 12: e69-e100.

2. Varma N, Epstein AE, Irimpen A, Schweikert R, Love C. Efficacy and safety of automatic remote monitoring for implantable cardioverter-defibrillator follow-up: The Lumos-T Safely Reduces Routine Office Device Follow-up (TRUST) trial. Circulation 2010; 122: 325-332.

3. Guedon-Moreau L, Lacroix D, Sadoul N, Clementy J, Kouakam C, Hermida JS, et al. A randomized study of remote follow-up of implantable cardioverter defibrillators: Safety and efficacy report of the ECOST trial. Eur Heart J 2013; 34: 605-614.

4. Parthiban N, Esterman A, Mahajan R, Twomey DJ, Pathak RK, Lau DH, et al. Remote monitoring of implantable cardioverterdefibrillators: A systematic review and meta-analysis of clinical outcomes. J Am Coll Cardiol 2015; 65: 2591-2600.

5. Saxon LA, Hayes DL, Gilliam FR, Heidenreich PA, Day J, Seth $\mathrm{M}$, et al. Long-term outcome after ICD and CRT implantation and influence of remote device follow-up: The ALTITUDE survival study. Circulation 2010; 122: 2359-2367.

6. Hindricks G, Taborsky M, Glikson M, Heinrich U, Schumacher B, Katz A, et al. Implant-based multiparameter telemonitoring of patients with heart failure (IN-TIME): A randomised controlled trial. Lancet 2014; 384: 583-590.

7. Mahoney FI, Barthel DW. Functional evaluation: The Barthel Index. Md State Med J 1965; 14: 61-65.

8. Charlson ME, Pompei P, Ales KL, MacKenzie CR. A new method of classifying prognostic comorbidity in longitudinal studies: Development and validation. J Chronic Dis 1987; 40: 373-383.

9. Sweeney MO, Hellkamp AS, Ellenbogen KA, Greenspon AJ, Freedman RA, Lee KL, et al. Adverse effect of ventricular pacing on heart failure and atrial fibrillation among patients with normal baseline QRS duration in a clinical trial of pacemaker therapy for sinus node dysfunction. Circulation 2003; 107: 2932-2937.

10. Morgan JM, Kitt S, Gill J, McComb JM, Ng GA, Raftery J, et al. Remote management of heart failure using implantable electronic devices. Eur Heart J 2017; 38: 2352-2360.

11. Mabo P, Victor F, Bazin P, Ahres S, Babuty D, Da Costa A, et al. A randomized trial of long-term remote monitoring of pacemaker recipients (the COMPAS trial). Eur Heart J 2012; 33: $1105-1111$.

12. Laurent G, Amara W, Mansourati J, Bizeau O, Couderc P, Delarche $\mathrm{N}$, et al. Role of patient education in the perception and acceptance of home monitoring after recent implantation of cardioverter defibrillators: The EDUCAT study. Arch Cardiovasc Dis 2014; 107: 508-518.

\section{Supplementary Files}

Please find supplementary file(s);

http://dx.doi.org/10.1253/circrep.CR-20-0071 